\title{
Modelling on-demand preprocessing framework towards practical approach in clinical analysis of diabetic retinopathy
}

\author{
Prakruthi Mandya Krishnegowda ${ }^{1}$, Komarasamy Ganesan ${ }^{2}$ \\ ${ }^{1}$ Department of Computer Science and Engineering, Jain (Deemed to be University), Bengaluru, India \\ ${ }^{2}$ School of Computing Science and Engineering, VIT Bhopal University, Sehore, India
}

\begin{tabular}{l} 
Article Info \\
\hline Article history: \\
Received Jan 1, 2021 \\
Revised Jul 15, 2021 \\
Accepted Aug 4, 2021 \\
\hline
\end{tabular}

Keywords:

CLAHE

Diabetic retinopathy

PSNR

\begin{abstract}
Diabetic retinopathy (DR) refers to a complication of diabetes and a prime cause of vision loss in middle-aged people. A timely screening and diagnosis process can reduce the risk of blindness. Fundus imaging is mainly preferred in the clinical analysis of DR. However; the raw fundus images are usually subjected to artifacts, noise, low and varied contrast, which is very hard to process by human visual systems and automated systems. In the existing literature, many solutions are given to enhance the fundus image. However, such approaches are particular and limited to a specific objective that cannot address multiple fundus images. This paper has presented an on-demand preprocessing frame work that integrates different techniques to address geometrical issues, random noises, and comprehensive contrast enhancement solutions. The performance of each preprocessing process is evaluated against peak signal-to-noise ratio (PSNR), and brightness is quantified in the enhanced image. The motive of this paper is to offer a flexible approach of preprocessing mechanism that can meet image enhancement needs based on different preprocessing requirements to improve the quality of fundus imaging towards early-stage diabetic retinopathy identification.
\end{abstract}

This is an open access article under the CC BY-SA license.

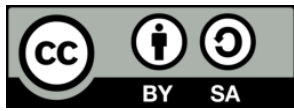

\section{Corresponding Author:}

Prakruthi Mandya Krishnegowda

Department of Computer Science and Engineering, Jain (Deemed to be University)

Bengaluru, India

Email: mkprakruthi.1012@gmail.com

\section{INTRODUCTION}

Diabetic Retinopathy refers to a vision disorder caused by a metabolic disease called diabetes, which causes vision damage ranging from blurred vision to complete blindness in diabetic patients [1], [2]. Diabetic retinopathy (DR) progresses in three stages a minor, moderate, and advanced stage, causing retinal ischemia and complete blindness, which is characterized by the appearance of symptoms such as microaneurysms and exudates and many other pathological features [3]. Effective screening and diagnosis are required in ophthalmology clinical analysis to detect DR and suitable treatment at an early stage. To date, lots of medical imaging techniques are available in the biomedical field, among which fundus imaging is the best available imaging modality in ophthalmology to carry out diagnosis and identification of DR [4], [5]. However, one of the major issues encountered in detecting blood vessels and diagnosing pathological signs is the poor image quality due to poor contrast, uneven illumination, and noise inclusion during the fundus image acquisition process [6]. Therefore, a preprocessing mechanism is needed for executing effective image segmentation and an accurate disease identification process. As a primary step in preprocessing, image enhancement is carried out to improve the contrast and vividness of the features in the fundus images [7]. Changes in the elements and textures of objects in the enhanced fundus images provide an essential biomarker for carrying out comprehensive clinical analysis of the region of interest (ROI) towards pre- 
evaluation of patient and diagnosis of DR [8]. There isa range of preprocessing approaches given in the literature for enhancing the quality of the fundus images. Gamma correction and histogram-based techniques are extensively adopted to improve fundus images' visual characteristics due to their admirable performance [9], [10]. Although there is various availability of preprocessing medical objects, there is less evidence of performing effective preprocessing towards the retina. Therefore, this paper presents a discussion of a computational model of preprocessing aiming towards DR. The manuscript's organization is as follows: Section 2 discusses the existing studies in the form of the study background, section 3 highlights the research problems, while Section 4 briefs about proposed methodology in terms of solution. Section 5 discusses the proposed algorithm while section 6 discusses the result and discussion, and section 7 discusses the conclusion of the study.

\section{RELATED WORK}

This section discusses existing studies towards preprocessing. Xiao et al. [11] adopted a gamma correction mechanism for the fundus image enhancement for the automatic detection of DR extracting the green component of the fundus image and determined the histogram curve slope. Mouzai et al. [12] presented a fuzzy-based gamma correction mechanism for brightness preservation. According to the study of [13], it is found that histogram-based techniques, especially histogram equalization (HE) and contrast limited adaptive histogram equalization, are mainly used to increase contrast medical images. However, contrast limited adaptive histogram equalization (CLAHE) is introduced as an improvised version of HE that uses a clip limiting mechanism, which reduces the over brightness and provides better enhancement results [14]. Yadav et al. [15] mentioned HE is subjected to amplification of noise and over enhancement factor. Shamsudeen et al. [16] introduced an improved HE mechanism to adjust brightness and noise elimination. The adoption of a joint approach of gamma correction and CLAHE is found in the study of Zhou et al. [17]. In this work, the luminance gain matrix is constructed by applying a gamma correction mechanism in the HSV color space for each color component of the input image.

Further, the secondary level of contrast adjustment is carried out in the channel, where CLAHE is applied over the LAB color space. This technique has demonstrated an intelligent approach of gamma correction and CLAHE together to enhance the contrast of the fundus image. Apart from using contrast adjustment techniques, filtering mechanisms such as mean and median filtering are considered to eliminate the fundus image noises. A combination of noise filters and CLAHE is considered in the study of Kumar et al. [18]. This study uses a fuzzy-based median filter and block matching algorithm to perform noise elimination. CLAHE and the modified HE technique enhance the contrast of the filtered image. In a similar direction, the work done by Sonali et al. [19] used a combination of various noise filters such as mean, median, Gaussian, and CLAHE are then applied to the denoised image color components. Adopting the haze removal algorithm is also seen in Vinodhini et al. [20] to adjust un-even illumination in fundus image pixel intensity. The edge is smoothed by using a filtering mechanism. Reddy et al. [21] presented an integrated enhancement algorithm to perform optimal enhancement of retinal images. The authors have used a flight firefly optimization approach with adaptive gamma-corrected and texture HE framework for improving overall visual quality. However, this study witnesses computational complexity as it performs a multi-level computation for different techniques. Fraz et al. [22] presented a survey of existing methods for extracting the retinal vessel. Mohanty et al. [23] and Dai et al. [24] carried extensive investigation and analysis of the fundus image screening and automatic DR identification diagnosis. Khan et al. [25] presented a review work on the fundus imaging techniques towards the precise extraction of blood vessels. Lin et al. [26] carried a systematic analysis of retinal images for the automatic screening of the DR.

\section{PROBLEM STATEMENT}

The research problems based on the above-related work are analyzed as follows:

- Irrespective of extensive research towards DR using fundus images while focusing on addressing enhancement issues, there are fewer standard models.

- Usage of image enhancement techniques is either carried in the limited scope of preprocessing operation. In addition, some are associated with a computational complexity that doesn't meet the requirement of effective diagnosis and DR screening.

- The existing enhancement mechanism is carried out highly recursive, which a simple and time-efficient implementation design needs approach for fundus images.

- Also, there is less availability of joint mechanisms to address several enhancement problems in a single module. Actually, the enhancement is carried out to specific objectives, mainly to research work. The 
literature lacks a comprehensive set of preprocessing approaches to meet all image enhancement requirements in a single deployment scenario.

Therefore, the problem statement for the proposed system can be stated as "Developing a computational model that can provide on-demand preprocessing approaches to address multiple enhancement problems associated with fundus image is a challenging task."

\section{PROPOSED SOLUTION}

The proposed research study intended to present a unique solution to address different problems related to image quality within a single set of computational models. The current research work presents the computational framework modeling, which offers on-demand preprocessing services to carry out comprehensive enhancement operations over fundus images. The on-demand preprocessing is meant to facilitate a variety of image quality improvement mechanisms in a single application, with an advantage over multiple preprocessing requirements as per the visual characteristics of the fundus imaging. The proposed framework integrates different preprocessing algorithms. The selection of suitable enhancement as a practical solution is based on evaluating each preprocessing technique concerning a particular input fundus image. The performance evaluation is carried based on the enhanced image statistics and quality metrics such as peak signal-to-noise ratio (PSNR) and Brightness as a performance indicator for each preprocessing technique. The architecture of the on-demand preprocessing framework is depicted in Figure 1.

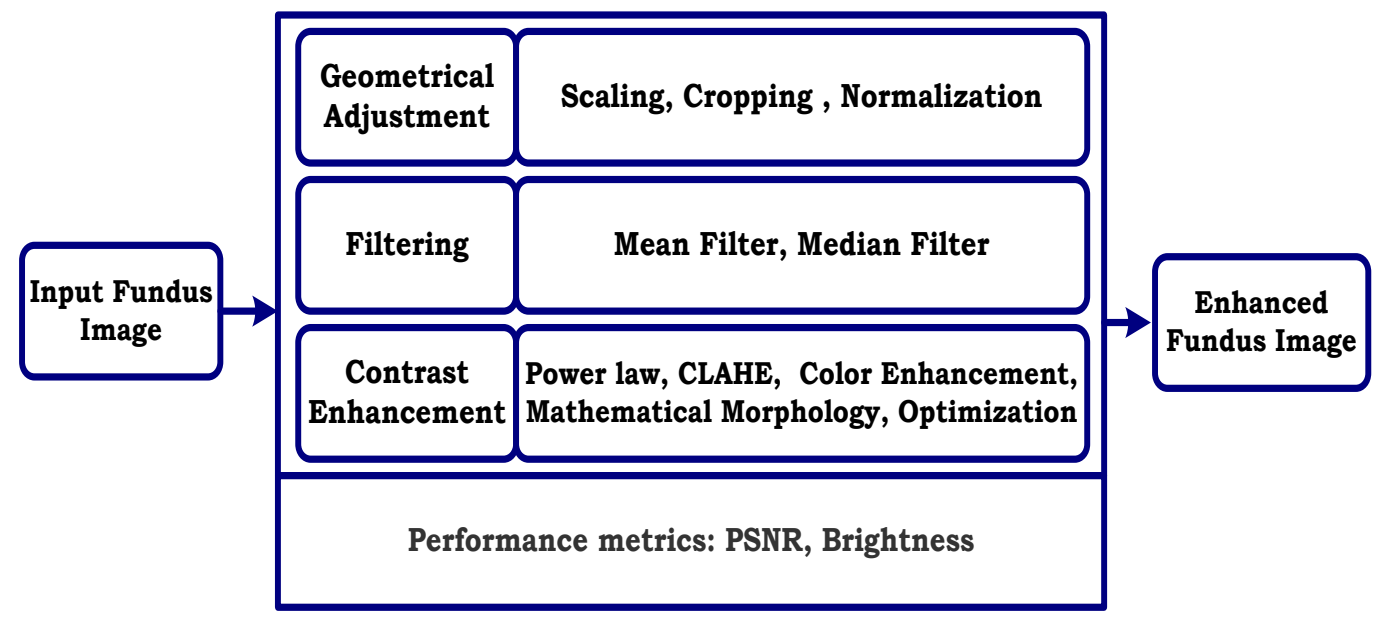

Figure 1. Outline of the proposed pre-processing techniques and evaluation model

Figure 1 depicts the architecture of the proposed on-demand preprocessing framework, which incorporates different preprocessing techniques such as interpolation, cropping, and normalizing to address geometrical deformations. In addition, mean filtering, median filtering, and recursive filtering-based preprocessing mechanisms are evaluated to handle a higher noise level in the input fundus image. Apart from noise elimination and geometric adjustment, the study also evaluates various contrast enhancement techniques such as power law, CLAHE, low-light color enhancement, mathematical morphology, and particle swarm optimization (PSO)-based optimization to address low contrast factors and uneven illumination. This research study aims to offer a novel and versatile image preprocessing model that automates clinical analysis where time and accuracy are crucial factors. Thus, it allows the physician to choose preprocessing techniques flexibility to perform a significant enhancement over image for early detection of DR. The considerable contributions of the proposed research work are as follows:

- The proposed on-demand pre-processing framework provides a potential solution to address several issues often encountered in the image enhancement process. It can also be used interchangeably depending on the input image's visual characteristics.

- The evaluation of the pre-processing techniques integrated into the proposed system is carried based on the output response and the image statistics.

- It also provides cost-effectiveness and time efficiency in clinical analysis. In addition, physicians can conduct more profound research of fundus imaging by selecting different pre-processing mechanisms integrated into the proposed system. 
- In addition, the proposed framework also provides the benefit of optimization in the enhancement process using a swarm-based stochastic approach with the flexibility of fine-tuning its parameter for a better analysis of the image based on the particular requirements.

\section{THE PROPOSED ALGORITHM}

In this section, the algorithms are discussed used in the implementation of the proposed on-demand preprocessing mechanism. The prime concern behind the implementation of this algorithm is that different fundus images come with a wide variety of problems. These problems need to be addressed in the preprocessing phase as a prerequisite for human analysis and the automation processes in the clinical research towards disease identification and patient treatment. Therefore, the proposed system focuses on three different categories of preprocessing issues and presents potential solutions that work in an integrated way to offer a comprehensive enhancement of the fundus image.

\subsection{Algorithm for geometric adjustment}

The geometric adjustment process refers to rectifying distortions associated with image shape, object reflection, scale, orientation, and normalization. The proposed research study considers three geometric-related issues; i) image up scaling and downscaling ii) region of interest mining, and iii) grayscale normalization. The fundus image photographer adopts different field views (FoV) to achieve fundus imaging. Thus, the acquired images are variable in size according to diagnostic requirements. The algorithm for implementing the scaling technique is described as follows:

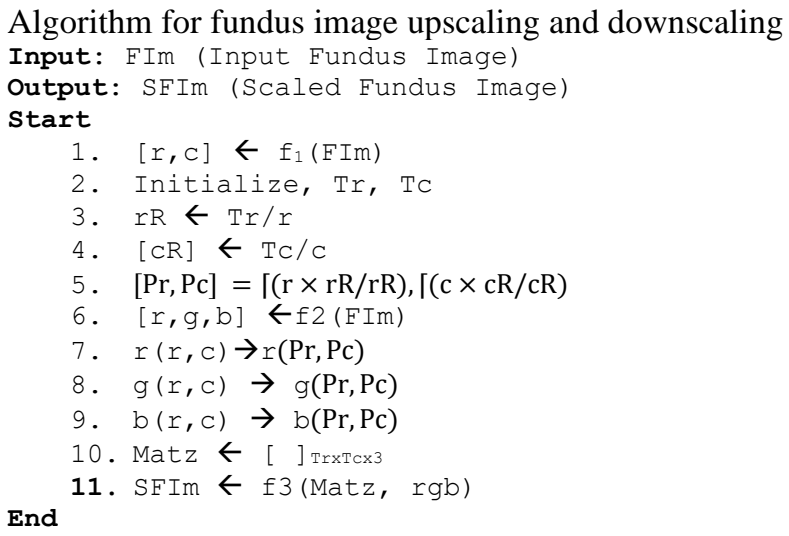

In the first step of the algorithm, the system uses a function f1() over the input image to get the size of the input fundus image FIM as the number of row $r$ and column $\mathrm{c}$ (line1). In the second step, a variable target row (Tr) and target column (Tc) are initialized to define the new size of the image (line2). To perform scaling, the algorithm computes a scaling ratio $\mathrm{rR}, \mathrm{cR}$ by dividing the target image size by the size of the original image in a row-wise and column-wise manner (Line3 \& Line4). These scaling ratios are then used to compute Pr and Pc's scaling positions for the pixel replication process (Line5). The (1) exhibits the computation of scaling positions as:

$$
[P r, P c]=\lceil((r \times r R) / r R),\lceil((c \times c R) / c R)
$$

where $\mathrm{Pr}$ is the row-wise scaling position and $\mathrm{Pc}$ is the column-wise scaling position, $\Gamma$ is the function that denotes rounding towards the nearest integer. The algorithm defined a function f2() to extract the color components RGB from the input image to perform a pixel replication process in each color component. The computed scaled row position Pr and column scaled position Pc (Line6-9). Further, a null matrix is constructed whose all entries are zeros with the size of the target image (Line 10). Anew rescaled image is then obtained in the next step of the algorithm using function $\mathrm{f3}()$, which performs sampling of all three color components into the null matrix that finally constructs the new image (SFIm) with the size of $\mathrm{Tr}$ and $\mathrm{Tc}$ (Line11). The next operation in the geometric adjustment is the extraction of the object in the image under consideration. The study considers this part a significant function to include in the proposed system. It facilitates the physician to work on only a specific part rather than analyzing the whole image. A cropping mechanism is adopted to perform RoI extraction, which also acts as a primary segmentation mechanism to perform image analysis for eye-related disease identification. The normalization of input fundus image is carried out in grayscale representation under a range of even distribution of pixels as in (2): 


$$
\mathrm{NFIm}=((F I m-\mathrm{pmin}) \times 255) /(\text { pmax }- \text { pmin })
$$

where NFIm is the normalized input, FIm is the normalized imaged, pmin, and pmax is the minimum and maximum scale of the input image FIm. This also performs a similar operation of denoising in the input image.

\subsection{Algorithm for denoising}

The proposed framework considers three different filtering operations, such as a mean, median, and combined mean-median filter to de-noise the image and generates an enhanced image as an output. The algorithm for noise filtering in the image is described as follows:

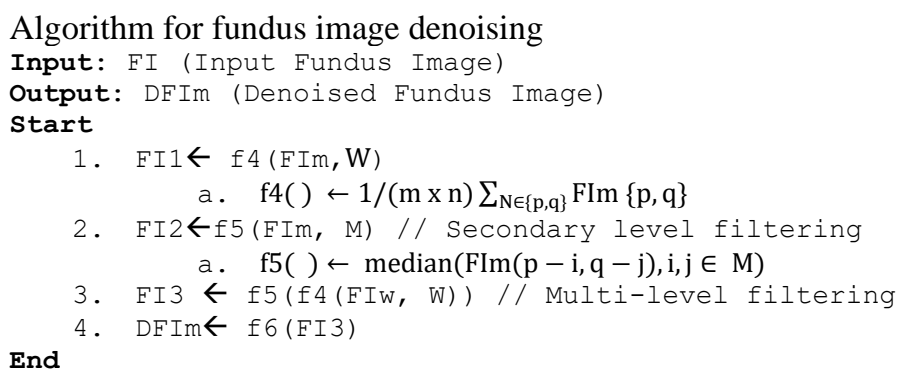

The first step of the proposed algorithm is mainly subjected to the mean filtering process FI1. An averaging function $\mathrm{f} 4($ ) is used to perform the filtering process using linear operation with $\mathrm{F}$ (filter mask $=\mathrm{m} \mathrm{x}$ $\mathrm{n}$, where $\mathrm{n}=3$, and $\mathrm{m}=3$ ). The function $\mathrm{f} 4()$ determines the mean value of the FIm $(\mathrm{p}, \mathrm{q})$ with the $\mathrm{W}$ centered at point $p$, q pixel coordinate of FIm (Line1). The filtered image (mFI) at point $(p, q)$ can be given as in (3):

$$
m F I=1 /(m \times n) \sum_{N \in\{p, q\}} F \operatorname{Im}\{p, q\}
$$

where $\mathrm{mFI}$ is the filtered image and $\mathrm{W}$ is the filter mask or window as a square matrix of size $\mathrm{m} \mathrm{x} \mathrm{n,} \mathrm{N} \mathrm{refers}$ to the neighborhood values belonging to the set of pixel coordinate $\mathrm{p}, \mathrm{q}$ in the square matrix $\mathrm{F}$.

This operation is executed using convolution matrix, where each coefficient considered with the value of $1 / F$. This operation is computationally efficient and effective in smoothing the image's visual quality suffering from the blurriness. However, if the input fundus image is subjected to a higher noise or random noise level, then the input image processed with mean filtering lacks the edge details in the resulting output. The median filtering FI2 is considered in the proposed system to eliminate random noise issues in the input image. Similarly, a median filter is applied using function f5(), which executes non-linear operation considering computation of the median value of the FIm with filter mask M (Line2). In this process, the coordinates points of a pixel in the mask $\mathrm{M}$ are arranged based on the grayscale values, and the median value of $\mathrm{M}$ is used to eliminate the noisy values. It restores the noise-free pixel values in the $\mathrm{M}$. The output image achieved by non-linear enhancement operation described as (4):

$$
\operatorname{mdFI}(p, q)=\operatorname{median}(F I m(p-i, q-j), i, j \in M)
$$

where $\mathrm{mdFI}$ is the output denoised fundus image, $M$ is the mask of size $n \times m$, where $m, n=3 \times 3$.

This technique is effective in the elimination of random noise, blurriness in the fundus images. However, the proposed study also implemented a combined approach of mean, median, and first-order recursive filtering approach to perform a multi-level filtering process to eliminate higher-level noise in the input image (Line3). In this process, the outcome obtained (FI1) from the mean filtering approach is taken as input for the median filtering approach of mask M size 5x5. Output image FI3 is then processed with a firstorder recursive filter [27]. A recursive filtering function f6() is used with an input argument FI3 (output of combined mean and median filter).

\subsection{Algorithm for contrast enhancement}

Various image enhancement techniques implemented in the proposed system to contrast adjustment and brightness preservation in the input image are discussed in this section. Power-law-based enhancement is widely used in the existing literature to improve the contrast of images based on the gamma correction factor.

Algorithm for fundus image Contrast enhancement using power-law transforms

Input: FI (Input Fundus Image)

Output: EFI (Enhanced Fundus Image), b2 (brightness EFI), s2 (sharpness EFI)

Modelling on demand preprocessing framework towards ... (Prakruthi Mandya Krishnegowda) 


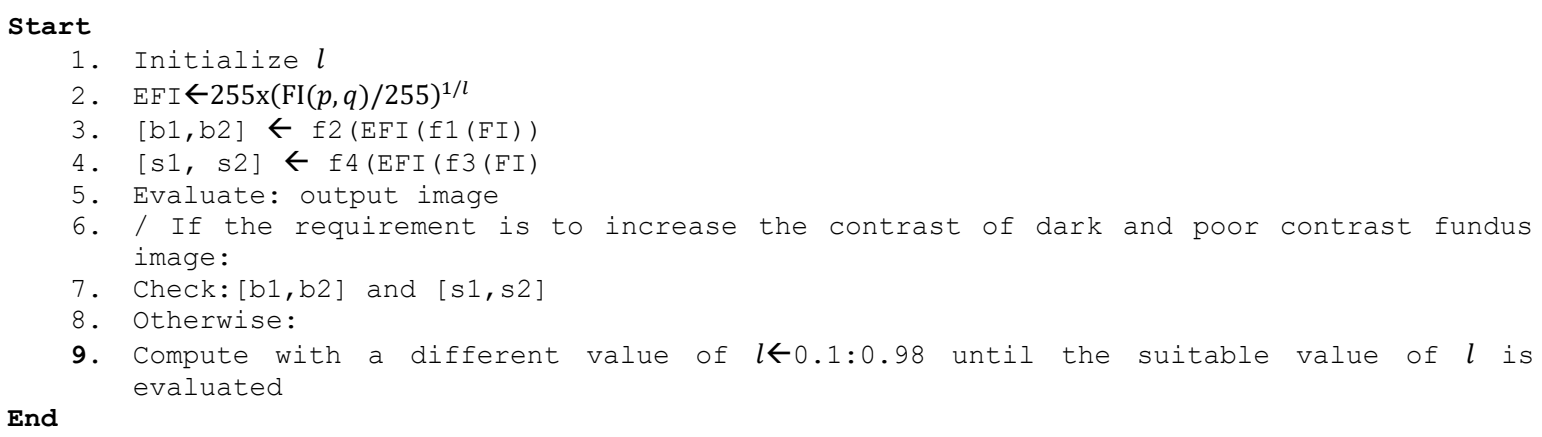

The first step of the algorithm is to initialize the variable $l$ attribute for contrast correction (Line1). Then, the contrast correction for given image enhancement is carried using numerical expression mentioned in the algorithm, which refers to a non-linear operation that maps the un-even illumination value in the input image to the uniform luminance in the output space using correction factor $l$ followed by power-law transformation (line 2). In the next step (Line3), the algorithm computes the brightness value b2 and b1 for both enhanced image using function $\mathrm{f} 2()$ and input image using function f1(). Similarly, the quantified sharpness s1 and s2are also computed for both input image and output image using function f3() and f4()respectively for the particular value of the correction factor attributel Line $4 \& 5$. However, the requirement is to increase the image's contrast; therefore, the system evaluates both quantified sharpness and brightness to justify the need for FI enhancement using this technique (Line 7\&8).However, if the user is not satisfied, then the same steps will be followed with different values of $l$ within the range of 1:0.98. In addition, the value of lcan not be considered one because the power law transformation function performs liner operation, which means no mapping of output space with contrast corrected values. The enhancement requirement also is justified with the histogram analysis. CLAHE has been an extensively adopted mechanism for enhancing the contrast of fundus imaging and other medical imaging modalities. In CLAHE, the image is divided into evenly distributed blocks, and the histogram of each block is determined. Before computing the global histogram and contrast intensity, the local histogram of each block is clipped using a clipping limit (cL) defined based on the user requirement. For example, for input image FI with $\mathrm{m}$ row and $\mathrm{n}$ column, the cL is given by (5):

$$
c L=\left\{\begin{array}{c}
\frac{1}{c F(m n)} \\
\frac{c}{S}
\end{array} \text { if } \frac{c f(m n)}{S}<1\right.
$$

where $\mathrm{cF}$ is the contrast factor, $\mathrm{S}$ is the histogram slope.

The clipped histograms are normalized, and the cumulative distribution of each block is computed based on the distribution parameter. The adjacent blocks are united using bilinear interpolation to eradicate unwilling boundaries. The advantage of using CLAHE is that it overcomes the issues associated with over brightness. The fundus images are often captured in low-lightening conditions. As a result, images are generated with low contrast, high noise, and haziness. The study considered a low-light color image enhancement technique to increase brightness and sharpness in the images captured in a low-lighting environment. The algorithm for low-lighting color enhancement is discussed as follows:

Algorithms for fundus image contrast enhancement using power-law transform

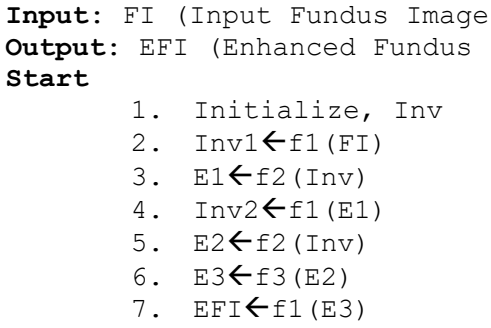

In the first line of the algorithm, a variable Inv is initialized to compute an inverted image (Line1). A function $\mathrm{f1}()$ is used to compute the complement of input FI, and the output is stored as a first-level inverted image (Line 2). The complement of the image is carried out using a formula, i.e., 255-FI, where 255 is the maximum color intensity value. In the following process, a function f2() is applied over Inv1 (inverted 
image) to enhance the image using the haze removal algorithm (Line3). To improve enhancement output, a similar process is repeated to perform a secondary level of enhancement. Therefore, a function f1() is again applied to the E1, an enhanced image computed in the previous step to compute complement. The secondary level of enhanced image E2 is computed using a similar function f2() that calls the haze removal algorithm (Line4 \& Line5). The improved image E2 is further subjected to the guided filter [28], using function f3(), which provides edge smoothed image E3 as the final enhanced image. Finally, the enhanced inverted image is restored into its original color state as enhanced fundus image (EFI) enhanced fundus image using function f1(). The reason behind adopting the haze removal-based low-light color fundus image algorithm is to explore its effectiveness towards benefitting enhancement on dark color FI images. The proposed system also offers a mechanism for identifying one of the most significant features known as exudates using the mathematical morphological mechanism. The algorithmic steps are given as follows:

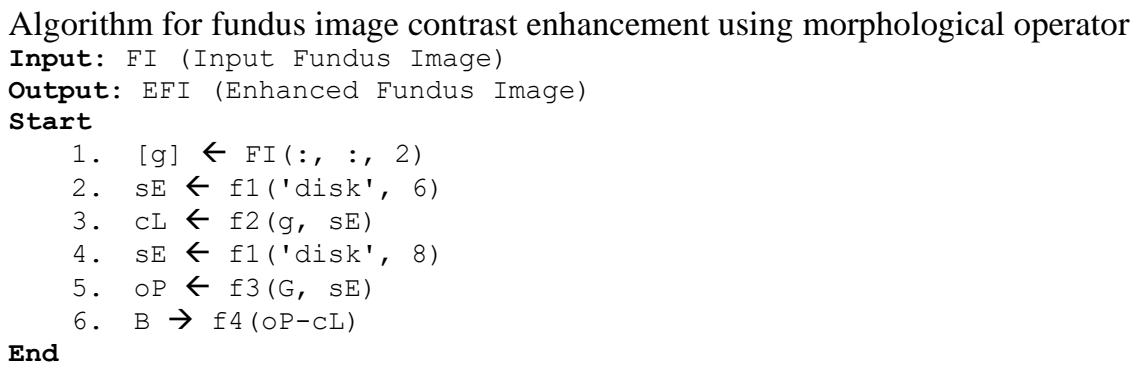

The algorithm takes input fundus image FI and, after a morphological operation, provides extraction exudates in a contour plot. The first step of the algorithm is to extract the green ' $\mathrm{g}$ ' color channel from FI to initiate the morphological operation (Line1). A disk shape like structuring element $(\mathrm{sE})$ is constructed using function f1() with radius length of pixels (Line2) to perform closing operation 'cL' followed by morphological bottom hat operation function f2() given as (6):

$$
g \cdot s E=(g \oplus s E) \ominus s E
$$

where $\mathrm{g}$ is the green color channel extracted from the FI, $\mathrm{sE}$ is the structuring element, · closing operator, $\oplus$ dilation operator and $\ominus$ is the erosion operator.

In the bottomhat operation, the outcome from the above numerical expression is subtracted from the input image (Line3). This process provides a mechanism of vessel extraction obtained in the form of a low grayscale level object. In the next step, the algorithm again creates a structuring element using a similar function to initiate opening operation followed by tophat morphological (Line3 and Line4). The top that function $\mathrm{f} 3($ ) extracts a bright region with a higher intensity localization. This operation is given as (7):

$$
g \circ s E=(g \ominus s E) \oplus s E
$$

where $\circ$ is the morphological opening operator.

Further, the algorithm performs image binarization operation using function f4() and input argument of the difference between tophat and bottomhat values. This means the outcome achieved by the bottom hat is subtracted from the outcome achieved by the tophat process to extract precise exudates using a contour plot (Line6-7). The study explores the effectiveness of applying swarm-based optimization in the enhancement of the fundus image. The optimization method is based on the population-oriented search algorithm. The solution space is called a particle, and each particle has its fitness value Fv. The particle from the problem searches the solution space by optima in the iteration. Each solution is updated with the local best $(\mathrm{Pb})$ and global best $(\mathrm{gb})$ in each iteration, and further, it updated their velocity and position. This process continues until the requirement is fully satisfied or any error occurs. The algorithm steps are mentioned for proposed enhancement optimization.

The algorithm takes the input value as FI (fundus image) and generates EFI after executing an optimization algorithm (enhanced fundus image). First, the algorithm initializes the variables as max-Iter, Imax, Imin (maximum and minimum Inertial weight), v1, and v2 as acceleration coefficients, Psize population size (Line1). Then, the empty vector construction is carried out to store Fitness value Fv, local best $\mathrm{Pb}$, and global best gb position of the particles (Line2). The overall computation is carried on the grayscale image gFI. The size of the original image row ' $r$,' column 'c' is computed as a reference variable for the other computation process (Line3). For each iter, computation of variable I is carried out to determine the optimal set for parameters initialized. It also means that the parameters are subjected to each particle or 
solution space (Line4 \& Line5). Each particle size computes enhanced FI using function fx2() with an input argument of gFI, and initial particle position. Here the function fx2() refers to a transformation operation to enhance the input image. In the next step of the algorithm, discrete function fx3() with an input argument of the enhanced image, $\mathrm{m}$, and $\mathrm{n}$ is considered for computing fitness value Fv. The maximum value in the Fv max gets computed. The variable gb is computed as the maximum value of the $\mathrm{Pb}$ (Line6). The updating process for the of the particle new position $\mathrm{P}(\mathrm{x})$ and velocity $\mathrm{P}(\mathrm{v})$ is continued till the required criteria are matched (Line7 to 9), where w is the Inertial weight I and Pc previous velocity and Pl is the last location.

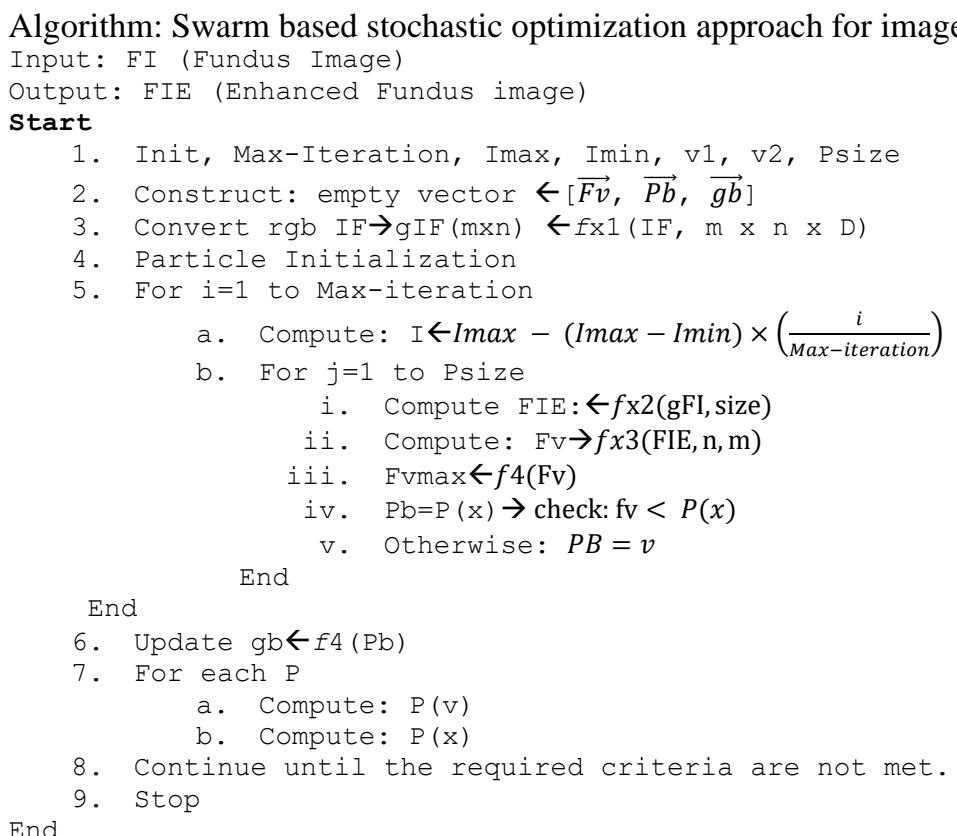

\section{RESULTS AND DISCUSSION}

The implementation of the proposed on-demand preprocessing framework is carried out on the numerical computing tool MATLAB. This section discusses the outcomes achieved by different preprocessing techniques for fundus image denoising and enhancement. Each method is evaluated over six different fundus images, where one is a dark fundus image, and the second one is a clear fundus image. The selection of enhanced images will be based on the visual outcome and quantitative analysis considering PSNR and quantified brightness. It can be seen that different preprocessing operation provides different outcomes in terms of enhanced image. The quantified analysis in terms of PSNR and Brightness is shown in Figures 2(a) and (b) and Figures 3(a) and (b). Figure 2(a) the analysis of enhancement techniques is carried out in PSNR, which shows better performance achieved by the CLAHE technique and other PSO optimization techniques. For linear evaluation, the clip limit (0.02) and gamma correction (0.89) values are considered fixed for all six images. For the input fundus images $(1,2,5$, and 6), gamma correction has shown higher PSNR. In the overall evaluation process, the low-light enhancement technique exhibited low PNSR compared to all other methods. The idea of considering a low-light enhancement image is to enhance the visibility of dark fundus images. The performance evaluation exhibited in terms of brightness shows that low-light enhancement techniques exhibit consistently higher performance. This evident the requirement of low-light enhancement techniques in the clinical analysis for the dark fundus images.

Figure 3(a) shows similar performance by the mean filtering approach and combined filtering approach. However, the performance exhibited by the median filter in both analyses PSNR and Brightness is a little less compared to other techniques. The reason can be multiple. The first one is the visual characteristic of input images, filter size, noise nature, and noise variance in the input image. The study has considered adding some additive Gaussian noise in the input image for the practical evaluation, which is then filtered by introduced filtering techniques. The performance of preprocessing techniques can be improved by fine-tuning their parameters like in the case of gamma correction (gamma value variation), CLAHE (i.e., clip-limit adjustment), and PSO (particle parameters).

The usage of image scaling, cropping, and normalization provides extra assistance for effective enhancement operations. Image scaling can be done for reducing or increasing the resolution of the images concerning computational complexity. The image cropping can also provide a better approach to visualizing 
the specific part of the input image. The normalization mechanism makes the distribution of image pixel intensity into a favorable range. A morphological approach is considered to detect the exudates' features from the input fundus image as a secondary preprocessing step. It can be regarded as the primary step towards the image segmentation process. The extraction of exudates is an effective bio-marker towards the detection of DR. The outcome of the morphological-based preprocessing operation is demonstrated in Figure 4.

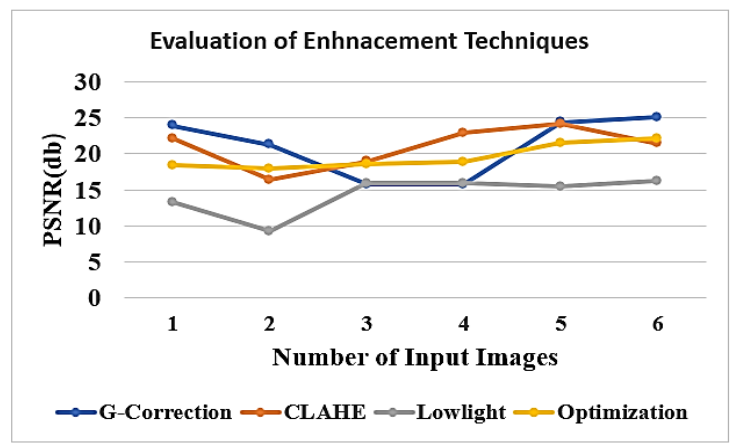

(a)

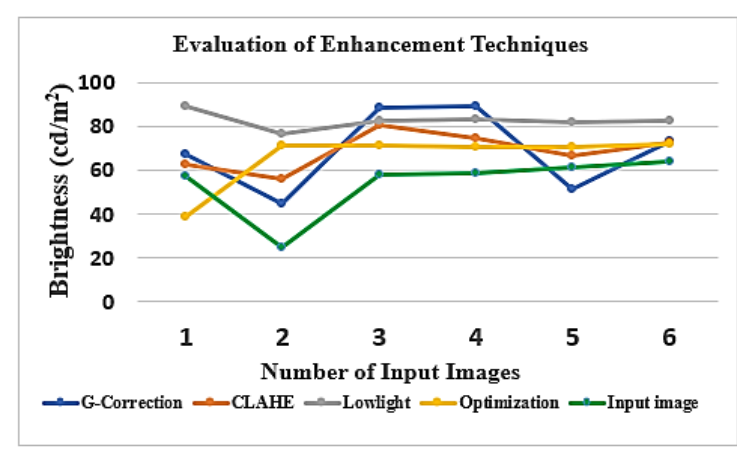

(b)

Figure 2. Evaluation of enhancement technique in terms of (a) PSNR, (b) brightness

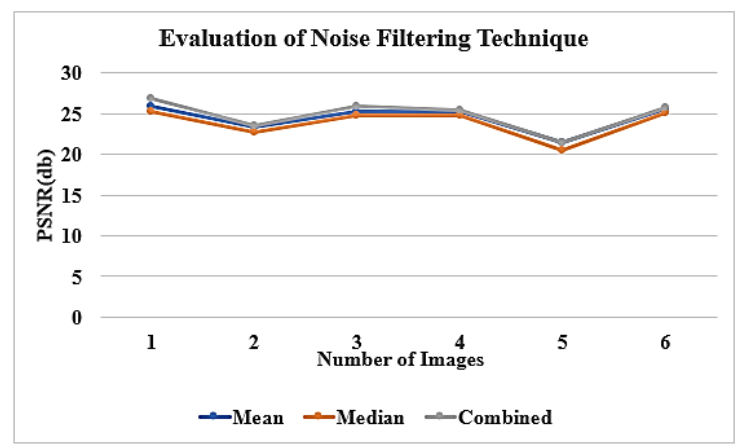

(a)

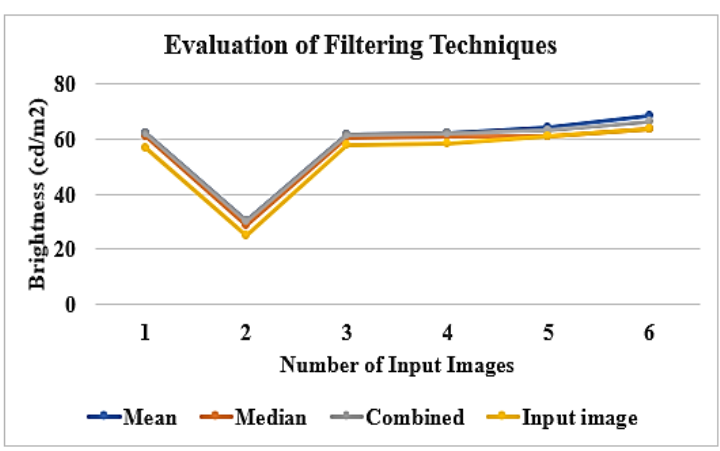

(b)

Figure 3. Evaluation of filtering technique in terms of brightness: (a) number vs. PSNR (db), (b) number of input images vs. brightness $(\mathrm{cd} / \mathrm{m} 2)$

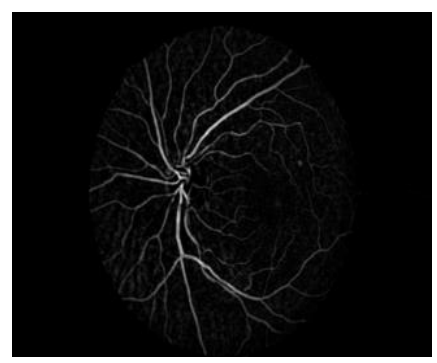

(a)

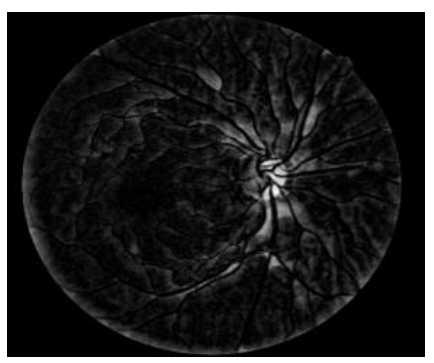

(b)

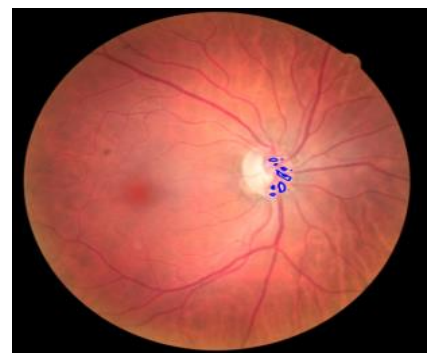

(c)

Figure 4. Primary segmentation of exudates using mathematical morphology: (a) morphological bottomhat, (b) morphological tophat, (c) exudates detection

Based on the performance evaluation, the proposed study concluded that selecting suitable enhancement techniques depends on the output image visual response, statistics, and quality metric. Therefore, it can be said that each preprocessing approach introduced in the proposed system is a complement to each other depending on the different preprocessing issues and enhancement required. The significant contribution of this study is that it offers the essentials of fundus image quality improvement, 
which is on-demand and flexible in an active environment to perform comprehensive enhancement over input image. Therefore, a collection of preprocessing packages with an interactive user interface is proposed, which offers a wide range of flexibility to enhance the fundus images.

\section{CONCLUSION}

In this paper, an on-demand preprocessing framework is modeled to address different enhancement issues and random noise associated with the fundus image. The proposed preprocessing framework integrated various techniques to address other problems categorized into three distinct parts: geometric-related problems, random noise and high-level noise, and enhancement-related problems. The study implemented an image scaling technique, RoI extraction, and image normalization to address geometric issues in the image. Similarly, different filtering techniques are adopted to address random and high-level noise. Contrast enhancement is improved using gamma correction, histogram analysis, low light color enhancement, morphological operator, and swarm optimization-based enhancement. Therefore, the proposed system can offer a flexible solution to overcome the limitations associated with the single image enhancement technique. The main objective of this work is to introduce a universal interface system that supports on-demand preprocessing operations with an option ofa wide range of preprocessing mechanisms. The user can efficiently perform appropriate enhancement over an image based on evaluating a particular medical image with different preprocessing options. The performance of each technique is validated based on qualitative and quantitative analysis. The proposed study can be extended to perform segmentation and diabatic retinopathy disease classification in future research work.

\section{REFERENCES}

[1] M. W. Stewart, Diabetic retinopathy: Current pharmacologic treatment and emerging strategies, Springer, ADIS, 2017, doi: 10.1007/978-981-10-3509-8

[2] A. R. Shah, and T. W. Gardner, "Diabetic Retinopathy: Research to clinical practice," Clinical diabetes and endocrinology, vol. 3, no. 1, pp. 1-7, 2017, doi: 10.1186/s40842-017-0047-y.

[3] J. Amin, M. Sharif, and M. Yasmin, "A review on recent developments for detection of diabetic Retinopathy," Scientifica, vol. 2016, 2016, Art. no. 6838976, doi: 10.1155/2016/6838976.

[4] B. J. Fenner, R. L. Wong, W. C. Lam, G. S. Tan, and G. C. Cheung, "Advances in retinal imaging and applications in diabetic retinopathy screening: a review," Ophthalmology and therapy, vol. 17, no. 2, pp. 333-346, 2018, doi: 10.1007/s40123-018-01537 .

[5] A. R. Sosale, "Screening for diabetic retinopathy is the use of artificial intelligence and cost-effective fundus imaging the answer?," International Journal of Diabetes in Developing Countries, vol. 39, pp. 1-3, 2019, doi: 10.1007/s13410-019-00729-y.

[6] A. Raj, A. K. Tiwari, and M. G. Martini, "Fundus image quality assessment: survey, challenges, and future scope," IET Image Processing, vol. 13, no. 8, pp. 1211-1224, 2019, doi: 10.1049/iet-ipr.2018.6212.

[7] F. Shao, Y. Yang, Q. Jiang, G. Jiang, and Y. Ho, “Automated quality assessment of fundus images via analysis of illumination, naturalness and structure," IEEE Access, vol. 6, pp. 806-817, 2017, doi: 10.1109/ACCESS.2017.2776126.

[8] N. R. Shenoy and A. Jatti, "Ultrasound image segmentation through deep learning based improvised U-Net," Indonesian Journal of Electrical Engineering and Computer Science (IJEECS), vol. 21, no. 3, pp. 1424-1434, 2021, doi: 10.11591/ijeecs.v21.i3.pp1424-1434.

[9] N. S. Zolkifli, A. Nazari, and M. M. Mustafa, "Retina blood vessel extraction based on Kirsch's template method," Indonesian Journal of Electrical Engineering and Computer Science (IJEECS), vol. 18, no. 1, pp. 318-325, 2020, doi: 10.11591/ijeecs.v18.i1.pp318-325.

[10] D. Devaraj and K. S. C. Prasanna, "Development of a hybrid framework to characterize red lesions for early detection of diabetic retinopathy," Indonesian Journal of Electrical Engineering and Computer Science (IJEECS), vol. 13, no. 3, pp. 962-973, 2019, doi: 10.11591/ijeecs.v13.i3.pp962-973.

[11] Z. Xiao et al., "Diabetic retinopathy retinal image enhancement based on gamma correction," Journal of Medical Imaging and Health Informatics, vol. 7, no. 1, pp. 149-154, 2017, doi: 10.1166/jmihi.2017.1998.

[12] M. Mouzai, C. Tarabet, and A. Mustapha, "Low-contrast X-ray enhancement using a fuzzy gamma reasoning model," Medical \& Biological Engineering \& Computing, vol. 58, pp. 1177-1197, 2020, doi: 10.1007/s11517-020-02122-y.

[13] K. G. Suma and V. S. Kumar, "A quantitative analysis of histogram equalization-based methods on fundus images for diabetic retinopathy detection," Computational Intelligence and Big Data Analytic, 2019, pp. 55-63, doi: 10.1007/978-981-13-0544-3_5.

[14] A. M. Reza, "Realization of the contrast limited adaptive histogram equalization (CLAHE) for real-time image enhancement," Journal of VLSI signal processing systems for signal, image and video technology, vol. 38, no. 1, pp. 35-44, 2004, doi: 10.1023/B:VLSI.0000028532.53893.82.

[15] S. K. Yadav, S. Kumar, B. Kumar, and R. Gupta, "Comparative analysis of fundus image enhancement in detection of diabetic Retinopathy," 2016 IEEE Region 10 Humanitarian Technology Conference (R10-HTC), Agra, India, 2016, pp. 1-5, doi: 10.1109/R10-HTC.2016.7906814.

[16] F. M. Shamsudeen and G. Raju, "Enhancement of fundus imagery," 2016 International Conference on Next Generation Intelligent Systems (ICNGIS), Kottayam, India, 2016, pp. 1 -5, doi: 10.1109/ICNGIS.2016.7854022.

[17] M. Zhou, K. Jin, S. Wang, J. Ye, and D. Qian, "Color retinal image enhancement based on luminosity and contrast adjustment," IEEE Transactions on Biomedical Engineering, vol. 65, no. 3, pp. 521-527, 2018, doi: 10.1109/TBME.2017.2700627.

[18] S. Kumar, S. Choudhary, R. Gupta, and B. Kumar, "Performance evaluation of joint filtering and histogram equalization techniques for retinal fundus image enhancement," 2018 th IEEE Uttar Pradesh Section International Conference on Electrical, Electronics and Computer Engineering (UPCON), 2018, pp. 1-5, doi: 10.1109/UPCON.2018.8597050. 
[19] S. S. Sonali, A. K. Singh, S. P. Ghrera, and M. Elhoseny, "An approach for denoising and contrast enhancement of retinal fundus image using CLAHE,” Optics \& Laser Technology, vol. 110, pp. 87-98, 2019, doi: 10.1016/j.optlastec.2018.06.061.

[20] A. C. Vinodhini, S. Sabena, and S. L. Ramesh, "A robust and fast fundus image enhancement by dehazing," International Conference On Computational Vision and Bio Inspired Computing, 2018, pp. 1111-1119, doi: 10.1007/978-3-030-41862-5_113.

[21] P. Reddy, S. H. Sujith, A. Kumar, L. K. Balyan, and H. N. Lee, "Retinal fundus image enhancement using piecewise gamma corrected dominant orientation based histogram equalization," 2018 International Conference on Communication and Signal Processing (ICCSP), 2018, pp. 0124-0128, doi: 10.1109/ICCSP.2018.8524518.

[22] M. M. Fraz et al., "Blood vessel segmentation methodologies in retinal images-a survey," Computer methods and programs in biomedicine, vol. 108, no. 1, pp. 407-33, 2012, doi: 10.1016/j.cmpb.2012.03.009.

[23] M. Mohanty, C. M. Mahapatra, and M. Mohanty, "A study on digital fundus images of retina for analysis of diabetic retinopathy," Advances in Machine Learning and Computational Intelligence, 2020, pp. 445-455, doi: 10.1007/978-981-15-5243-4_41.

[24] Y. Dai, C. Zhu, X. Shan, Z. Cheng, and B. Zou, "A survey on intelligent screening for diabetic retinopathy," Chinese medical sciences journal-Chung-kuo i hsueh k'o hsueh tsa chih, vol. 34, no. 2, pp. 120-132, 2019, doi: 10.24920/003587.

[25] K. B. Khan et al., "A review of retinal blood vessels extraction techniques: challenges, taxonomy, and future trends," Pattern Analysis and Applications, vol. 22, no. 3, pp. 767-802, 2019, doi: 10.1007/s10044-018-0754-8.

[26] J. Lin, L. Yu, Q. Weng, and X. Zheng, "Retinal image quality assessment for diabetic retinopathy screening: A survey," Multimedia Tools and Applications, vol. 79, pp. 16173-16199, 2019, doi: 10.1007/s11042-019-07751-6.

[27] E. S. Gastal and M. M. Oliveira, "Domain transform for edge-aware image and video processing," ACM Transactions on Graphics, vol. 30, no. 4, pp. 1-12, 2011, Art. no. 69, doi: 10.1145/2010324.1964964.

[28] K. He, J. Sun, and X. Tang, "Guided image filtering," IEEE Transactions on Pattern Analysis and Machine Intelligence, vol. 35, no. 6, pp. 1397-1409, 2013, doi: 10.1109/TPAMI.2012.213.

\section{BIOGRAPHIES OF AUTHORS}

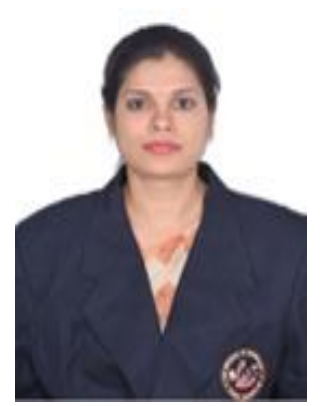

Prakruthi Mandya Krishnegowda (iD) If SC P is a Research Scholar in Jain University (Deemed to be University), Bengaluru. Currently, she is working as an Assistant Professor in Department of Computer Science and Engineering, S J B Institute of Technology, Bengaluru. She is having around 10 years of teaching experience. She has received M. Tech in Computer Science and Engineering from The Oxford college of Engineering, Bengaluru from Visvesvaraya Technological University, Belagavi in 2011. Her research interests include Health Care, Machine Learning, Information Security and Technology. She can be contacted at email: mkprakruthi.1012@gmail.com.

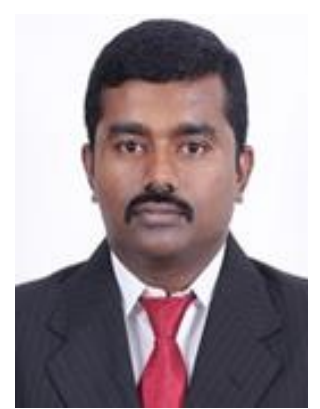

Komarasamy Ganesan (iD 8d sc P $\mathrm{P}$ working as Senior Associate Professor, in the School of Computing Science and Engineering, VIT Bhopal University, Kothrikalan, Sehore Madhya Pradesh, India . He has completed BE in 2003, ME in 2005 and Ph.D. awarded in 2014 in the area of Data Mining. He has 15 years of teaching experience. He has been awarded by prestigious IRDP Award 2019- "Dr. A. P. J. Abdul Kalam Life Time Achievement National Award" for the outstanding excellence and remarkable achievements in the field of Teaching, Research and Publications. He has published 30 Journals, presented 10 papers in International Conferences and 19 papers in National Conferences. His research domains are Data Mining, Machine Learning, and Internet of Things, Big data Analytics and Data Science. He can be contacted at email: gkomarasamy@gmail.com. 